\title{
Die Antinomien des Neofunktionalismus
}

\section{Eine Auseinandersetzung mit Jeffrey Alexander ${ }^{1}$}

\author{
Hans Joas \\ Institut für Soziologie der Universität Erlangen-Nürnberg, Kochstr. 4, D-8520 Erlangen
}

Zusa m m en fassung: Seit dem Beginn der achtziger Jahre unternimmt ein Kreis jüngerer amerikanischer Soziologen um Jeffrey Alexander energische und spektakuläre Versuche, durch Anknüpfung an das Lebenswerk von Talcott Parsons der Soziologie zu einem einheitlichen und umfassenden theoretischen Gerüst zu verhelfen. In diesem Aufsatz werden Alexanders Verdienste bei der Rechtfertigung allgemeiner soziologischer Theorie (insbesondere Handlungsund Ordnungstheorie) gewürdigt, zugleich aber Einwände gegen den Charakter von Alexanders Theorie erhoben. Die Prüfung von Alexanders metatheoretischen Überlegungen und seiner Interpretationen soziologischer Klassiker (Marx, Durkheim, Weber, Parsons) ergibt, daß Alexanders metatheoretischer Rahmen nicht geschmeidig genug ist, um die Problemlage der Klassiker wirklich zu rekonstruieren. Es werden Hinweise auf eine Handlungstheorie gegeben, die sich der Antinomie von Utilitarismus und Normativismus entzieht und damit theoretisch und interpretatorisch dem Erbe der soziologischen Klassiker angemessener ist.

Seit dem Beginn der achtziger Jahre unternimmt ein mehr oder minder homogener Kreis jüngerer amerikanischer Soziologen energische Versuche, durch Anknüpfung an das Lebenswerk von Talcott Parsons der Soziologie zu einem einheitlichen und umfassenden theoretischen Gerüst zu verhelfen. Ein nostalgischer Blick fällt dabei in die Jahrzehnte der unmittelbaren Nachkriegszeit zurück, in denen zumindest die amerikanische Soziologie Stabilität und Konsensus aufzuweisen schien. Heute dagegen wetteifern um einen Kernbereich einer theoriearmen quantitativen Forschungspraxis herum eine Vielzahl von Strömungen miteinander; sie erzeugen das Bild eines unübersichtlichen Pluralismus auch deshalb, weil ihr Wetteifer sich kaum mehr auf die argumentative Widerlegung der anderen Ansätze richtet, sondern lediglich in der Konkurrenz um knappe Mittel und Positionen Ausdruck findet. Dem Versuch einer neuen theoretischen Synthese stellen sich dabei ebenso wie einer Wiederbelebung des parsonianischen „orthodoxen Konsensus" eine Fülle von Schwierigkeiten in den Weg; zur Skepsis gegen Bedeutung und Möglichkeit allgemeiner Theorie überhaupt kommt das problemlose Einverständnis mit der pluralistischen Koexistenz einzelner Schulen hinzu und schließlich ein bis zur Unkenntlichkeit ver-

${ }^{1}$ Dieser Aufsatz erscheint in englischer Sprache in der Zeitschrift "Inquiry“ (Oslo). - Ich habe 1984 eine Rezension von Band 2 des vierbändigen Werkes (Alexander 1982/83) vorgelegt (Kölner Zeitschrift für Soziologie und Sozialpsychologie 36 [1984], S. 619-622); an einzelnen Stellen dieses Aufsatzes greife ich auf Formulierungen dieser Rezension zurück. stümmeltes, aus Gerüchten und tradierter Kritik zusammengesetztes Bild vom Werk Parsons'.

Am spektakulärsten hat Jeffrey Alexander versucht, dieser Schwierigkeiten Herr zu werden. Er hat in seinem vierbändigen Werk ,Theoretical Logic in Sociology“ (Alexander 1982/83) den Versuch unternommen, Parsons' erstes theoretisches Hauptwerk (die "Structure of Social Action“) in einer zeitgenössisch adäquaten Weise neu zu denken. Dieses Werk hat mittlerweile seine Fortsetzung im Versuch einer Soziologiegeschichte seit 1945 gefunden; hier wird der Zerfall der ParsonsHegemonie geschildert und unter dem Gesichtspunkt einer Selbstkritik des Parsonianismus gedeutet (Alexander 1987b). In einem von ihm herausgegebenen Sammelband hat Alexander das Programm einer neoparsonianischen, von ihm „neofunktionalistisch“ genannten Schule propagiert (Alexander 1985). Flankierende kleinere Beiträge verfolgten den Zweck, eine breitere Tendenz zur Parsons-Renaissance zu behaupten (Alexander 1984, 1986) oder die Verknüpfung von Theorieentwicklung und Klassikerinterpretation, die für sein mehrbändiges Werk typisch ist, methodologisch zu rechtfertigen (Alexander 1987a). In einer neuen Sammlung eigener Aufsätze (Alexander 1988) versucht Alexander, seine Theorie in der Anwendung auf empirische Bereiche zu erproben und zu modifizieren.

Alexanders theoretische Offensive trägt alle Züge einer professionspolitischen Strategie; dasselbe gilt allerdings für die meisten Reaktionen, die auf das Erscheinen seines Hauptwerks erfolgten. Der ehrgeizige und weitreichende Versuch, den seit Par- 
sons' Sturz verwaisten Thron selbst zu besteigen, mußte alle Arten von Gegnerschaft provozieren. Kaum jemals hatte ja ein Werk soziologischer Theorie, zumal ein von einem jungen und zuvor nicht sehr bekannten Autor verfaßtes, die interessierten Leser so überschüttet mit Vorschußlorbeeren erreicht. Prompt kam es, in einer ersten Runde der Kritik, im Rückstoß auf den hymnischen Ton der Vorankündigungen, zu vernichtenden Zweifeln am Sinn und der Originalität des ganzen Unternehmens und hämischen Verweisen auf stilistische und inhaltliche Abhängigkeiten vom Parsonsschen Vorbild. In einer zweiten Runde erschienen einige breite und tiefschürfende Kritiken an Alexanders Vorgehen und seinen Interpretationen soziologischer Klassiker (Burger 1986; Collins 1985; Porpora 1986; Turner 1985). Die entscheidende sachliche Frage aber, nämlich inwiefern Jeffrey Alexanders Arbeiten einen theoretischen Fortschritt in der Soziologie darstellen, wurde meist nur am Rande berührt. So reizvoll es sein könnte, die Auseinandersetzung um eine (behauptete) Parsons-Renaissance als Fallstudie professionspolitischer Konflikte zu schildern, soll hier doch ein anderer Weg gegangen werden. Es wird zu zeigen sein, daß Jeffrey Alexander zwar in verdienstvoller Weise die Notwendigkeit einer neuen theoretischen Synthese verficht, sich aber gleichzeitig der Mittel für die Erfüllung des selbstgesetzten Anspruchs teilweise beraubt. Der Fortschritt zur Einsicht in die Entwicklung einer umfassenden und synthetischen soziologischen Theorie wird bezahlt mit einer Abdichtung gegenüber den heutigen Möglichkeiten und Notwendigkeiten einer solchen Theorie. Dies soll an den Grundzügen von Alexanders Konzeption, an seiner Interpretation der Klassiker und am Zuschnitt seiner Soziologiegeschichte der Nachkriegszeit demonstriert werden.

Alexanders theoretische Konzeption läßt sich am schnellsten durch einen Vergleich mit dem Vorbild seines vierbändigen Werkes, eben Parsons' „Structure of Social Action“ (Parsons 1937) vergegenwärtigen. Parsons hatte in seinem Werk einen Ausweg aus dem Konflikt der beiden wichtigsten Schulen zeitgenössischer Ökonomie in den USA gesucht, nämlich der neoklassisch-grenznutzentheoretischen und der institutionalistischen. Während die Neoklassik mit dem Mittel einer Theorie rationalen Handelns eindrucksvolle Fortschritte zu einer theoriegeleiteten, quantifizierenden empirischen Erforschung eines Teilbereichs des sozialen Lebens vorweisen konnte, entsprach die institutionalistische Schule - ähnlich der historischen Schule der deutschen Nationalökonomie - besser dem
Bedürfnis nach einer Einbettung wirtschaftlicher Phänomene in die geschichtliche und gesellschaftliche Ganzheit. Für die Soziologie war diese Methodenkontroverse der Ökonomen insofern von zentraler Bedeutung, als auf den ersten Blick der von ihr beanspruchte Gegenstandsbereich mit dem der historisch-institutionalistischen Schulen sich zumindest teilweise überdeckte. Wollte die Soziologie aber nicht der Theorielosigkeit dieser Schulen verfallen, lag es näher, die umfassende Gegenstandsbestimmung dieser Schulen mit dem Theorieanspruch der Neoklassik zu kombinieren. Dies beinhaltete die Aufgabe, der Soziologie ein zur Theorie des rationalen Handelns analoges, von dieser Theorie aber eben unterschiedenes Fundament zu geben, d.h. die Grenzen der Theorie rationalen Handelns darzustellen und zugleich den ökonomischen Residualbereich nichtrationalen Handelns selbst positiv zu analysieren. Diese Problemlage hatte schon Max Webers Versuche zur Bestimmung von Handlungskategorien in den berühmten "Soziologischen Grundbegriffen" am Anfang von "Wirtschaft und Gesellschaft" gekennzeichnet $^{2}$. Für Parsons' Entwicklung vor seinem ersten Hauptwerk und für die Gedankenführung in diesem Werk selbst war diese Ausgangssituation entscheidend. Er erkannte zugleich die umfassendere, kulturdiagnostische Bedeutung dieser wissenschaftlichen Problemlage als Auseinandersetzung um die Reichweite und die Grenzen der gesamten utilitaristischen Moral- und Sozialphilosophie sowie - noch allgemeiner - einer liberalkapitalistischen Marktwirtschaft. Parsons begann sein Werk mit methodologischen Vorerörterungen, deren hauptsächliches Ziel es war, den ,analytischen" Status der Theorie des rationalen Handelns und möglicher Analogiebildungen herauszuarbeiten und damit substantialisierende Mißverständnisse $\mathrm{zu}$ verhindern, als sei die Theorie rationalen Handelns eine Theorie, welche die faktische Rationalität des sozialen Handelns ontologisch oder empirisch behaupte. Im Kern aber bestand Parsons' Werk aus weitgespannten Interpretationen der Schriften von Alfred Marshall, Vilfredo Pareto, Emile Durkheim und Max Weber. Diese

\footnotetext{
2 Anregungen von Göran Therborn folgend hat vor allem Simon Clarke (1982) diesen Hintergrund herausgearbeitet. Auf Parsons wendet dies neuerdings, wohl Anregungen von Donald Levine folgend, Charles $\mathrm{Ca}$ mic (1987) an. Meines Erachtens hat dieser vorzügliche Aufsatz nur eine Schwäche: er unterschätzt die Bedeutung Whiteheads für Parsons methodologisches Bewußtsein (dazu Wenzel 1985).
} 
Interpretationen wurden von der sogenannten "Konvergenzthese“ zusammengehalten, welche besagte, daß sich unter dem Gesichtspunkt einer soziologischen Handlungstheorie eine Konvergenz immanenter Überwindungsversuche des Utilitarismus (bei Marshall und Pareto) mit der sich vom Positivismus bzw. Idealismus ablösenden Begründung der Soziologie bei Durkheim und Weber nachweisen lasse. Diese Konvergenz war für Parsons nahezu ein empirischer Beweis für die Richtigkeit der sich herausschälenden Konzeption, da ja ohne wechselseitige Beeinflussung und auf dem Hintergrund sehr verschiedener nationaler Theoriemilieus die Übereinstimmung der Autoren nicht einfach als Ausdruck einer ideologischen Gemeinsamkeit aufgefaßt werden könne, sondern eher im Sinn einer „multiple discovery" als die voneinander unabhängige, aber gleichzeitige Lösung eines überbrachten Theorieproblems zu sehen sei. Diese Lösung bestand im Nachweis der normativen Dimension sozialer Ordnung und, damit eng verknüpft, in der Entwicklung des Kategorienrahmens einer ,voluntaristischen“ Handlungstheorie.

Wie verändert nun Alexander diese Konzeption bei der Konstruktion seiner eigenen Arbeit? Parsons' methodologischen Anfangskapiteln entspricht der erste Band, welcher auf dem Hintergrund des langen Reflexionsweges der postempiristischen oder "postpositivistischen" Wissenschaftstheorie die selbständige, weder auf Empirie noch auf Weltanschauung reduzierbare Ebene soziologischer Theoriebildung proklamiert und dies in der Benennung der beiden Fundamentalprobleme einer solchen Theorie, nämlich „Handlung“ und „Ordnung“, konkretisiert. Während die heutige Wissenschaftstheorie Alexanders Aufgabe erleichtert, erwarb Parsons nur durch die Stützung auf Whiteheads späte Wissenschaftsphilosophie eine gewisse Immunität gegen den Druck des logischen Positivismus. Die Nähe zu Parsons ist hier also durchaus mit einer größeren Explizitheit der Argumentation verbunden, als sie Parsons selbst erreichbar war. Die anschließenden Bände behandeln in ausführlichen Interpretationen das Werk von Marx, Durkheim, Weber und Parsons selbst. Der Zusammenhang mit der ökonomischen Kontroverse ist damit unerkennbar geworden; die Beschäftigung mit dem führenden Grenznutzentheoretiker Marshall ist ebenso wie die mit dem selbst vom Ökonomen zum Soziologen sich entwickelnden Pareto vollständig gestrichen. An deren Stelle tritt, formal gesehen, das Werk von Karl Marx, welches in Parsons' Konstruktion nur kurz und recht widersprüchlich einmal als Teil der utilitari- stischen Tradition, einmal als Teil der idealistischen Tradition auftauchte. Die Behandlung von Weber und Durkheim ist geblieben, wenngleich natürlich jeweils ein ganz anderer Kenntnisstand des Werkes dieser unumstrittenen Klassiker des Faches zugrundegelegt werden kann. Hinzugefügt wurde ein Band - der umfangreichste - über Parsons selbst als Klassiker. Diese Veränderungen mögen zunächst geringfügiger erscheinen als sie tatsächlich sind. Alexander beseitigt nämlich nicht nur den Hintergrund der innerökonomischen Kontroversen, sondern zugleich die Klammer, welche Parsons' monographische Interpretationen zusammengehalten hatte: die Konvergenzthese. Er erklärt diese nicht nur deshalb für falsch, weil in theoretischen Fragen der Sozialwissenschaften auch ein gleichzeitiger Wandel vieler Schulen noch als Ausdruck epochentypischer Konstellationen und nicht einfach als positiver Wissenschaftsfortschritt aufgefaßt werden könnte, - was nahegelegen hätte -, sondern weil er die These in der Sache für falsch hält. Die Konvergenz von Webers Theorie der Legitimität und des Charisma mit Durkheims Begriff des Heiligen zu erkennen, sei zwar eine theoretische Pionierleistung von Parsons gewesen; insgesamt aber lasse sich die Behauptung einer faktischen Konvergenz nicht halten und ergebe sich vielmehr nur aus den stillschweigenden Korrekturen, die Parsons an den intuitiv erkannten Schwachstellen der Klassiker vorgenommen habe.

Was aber hält die verschiedenen Interpretationen zusammen, wenn die Konvergenzthese hinfällig ist? Alexanders eigenes Konstruktionsprinzip liegt im Konflikt zwischen soziologischem Idealismus und soziologischem Materialismus und in der Überwindung dieses Konflikts in einem "multidimensionalen", "synthetischen" Ansatz. Für ihn stellen Marx und Durkheim einander ausschlieBende Vereinseitigungen dar. Der soziologische Materialismus des einen, der die angemessene Erfassung des Normativen und Kulturellen systematisch ausschließe, habe sein Gegenstück im soziologischen Idealismus des anderen, der zwar die entscheidenden Beiträge zu einer Theorie des Normativen geliefert, dieses aber nicht in die Welt der Bedingungen und Mittel überzeugend eingegliedert habe. Alexander versucht, an diesem Leitfaden die Entwicklung beider Denker nachzuverfolgen; dabei sieht er beide selbst in einem Konflikt zwischen den beiden Polen befangen. Zudem rekonstruiert er die Beiträge der wesentlichen Marxisten bzw. Durkheimianer aus dem Motiv heraus, die Folgeprobleme der Einseitigkeit des jeweiligen 
Meisters zu überwinden. Die Interpretation Max Webers im dritten Band dient dazu, den klassischen Versuch eines Auswegs aus dieser Antinomie darzustellen; über weite Strecken aber werden hier Abweichungen Webers vom Ideal einer „multidimensionalen“ Synthese kritisch aufgezeigt. Erst Parsons habe - allerdings keineswegs ohne Lapsus und Rückfälle - die begrifflichen Grundlagen einer solchen umfassenden Theorie vorgelegt.

Mit dieser Veränderung des Konstruktionsplans entzieht sich Alexander geschickt den vielfältigen Einwänden, die gegen Parsons' Konvergenzthese vorgebracht wurden; mit der Behandlung von Marx stopft er zugleich die auffallendste Lücke in Parsons' historischer Selbstverständigung. Sein Schachzug ist jedoch nicht ohne Probleme. Die neue Konstruktion ist ja nur dann überzeugend, wenn die Begriffe soziologischer Idealismus und Materialismus tatsächlich zentrale Probleme der soziologischen Theorie und des Lebenswerkes der behandelten Klassiker bezeichnen und wenn sich tatsächlich Webers und Parsons' Werke in einem vernünftigen Sinn als Versuch der Synthese von Marx und Durkheim auffassen lassen. Das ist aber keineswegs selbstverständlich. In mindestens drei Hinsichten möchte ich diese Konstruktion schon vor der Prüfung der Plausibilität der einzelnen Klassiker-Interpretationen in Frage stellen.

Der ewige Konflikt von Idealismus und Materialismus gilt heute nur noch in den Lehrbüchern des Marxismus-Leninismus als hinreichende Beschreibung für die Grundzüge der Philosophiegeschichte. Nun spricht Alexander freilich nicht vom Konflikt erkenntnistheoretischer Positionen, sondern von soziologischen Ansätzen. Hier bedeutet soziologischer Materialismus eine ganze Familie von Ansätzen wie Ökonomismus, Utilitarismus, Hedonismus, Behaviorismus, Darwinismus, Machiavellismus, denen die ungenügende Reflexion auf die zugrundeliegende Normativität gemeinsam ist; ihnen allen erscheint der Prozeß der Zielsetzung als bloß technischer Umgang mit äußerlichen Bedingungen $(I, 74)$. Demgegenüber besteht der soziologische Idealismus in einer einseitigen Berücksichtigung der normativen Dimensionen auf Kosten der situativen Bedingungen; sein Verdienst liegt allerdings in der Beharrung auf der Irreduzibilität des normativen Charakters menschlichen Handelns. Zumindest die Begriffswahl Idealismus und Materialismus für diese Problemlage ist sicher nicht besonders glücklich. Gemeint ist ja offensichtlich eher der Konflikt zwischen einer kantianischen und einer utilitaristischen Auffassung von Morali- tät und Sozialität. Wenn dies so bezeichnet worden wäre, wäre sofort klar geworden, da $B$ diese Charakterisierung des Grundproblems die soziologischen Klassiker nur insofern angemessen zu rekonstruieren erlaubt, als sie tatsächlich in diesem Spannungsverhältnis standen. Für Parsons und Weber kann man hier optimistischer sein als für Durkheim oder gar Marx. Eine kompliziert kritische Beziehung zur klassischen politischen Ökonomie oder zur Neoklassik, zu Spencers Soziologie oder zur utilitaristischen Moralphilosophie ist gewiß bei allen vier Denkern festzustellen. Parsons hat selbst der Rede von einem kantianischen Kern in seinem Werk Auftrieb gegeben, wenngleich damit nicht alle Zweifel ausgeräumt werden, es handle sich dabei eher um eine nachträgliche Selbststilisierung (Joas 1984). Für Weber und Durkheim ist der zeitgenössische Neokantianismus in Gestalt von Rickert oder Renouvier sicher nicht ohne formende Bedeutung gewesen; die Frage ist aber doch sehr, ob nicht die Auseinandersetzung mit Nietzsche einerseits, Bergson andererseits wesentlich wichtiger war. Um Marx zu verstehen, ist nicht nur Kant vonnöten, sondern die windungsreiche Geschichte des nach-kantischen deutschen Idealismus, die Romantik und das Linkshegelianertum. Behauptet werden soll hiermit nicht, daß Alexander nichts von der Bedeutung Hegels und Feuerbachs, Nietzsches und der Lebensphilosophie wüßte; seine Interpretationen kommen streckenweise durchaus darauf zu sprechen. Der Punkt ist vielmehr, daß die Kategorien, die er seinem Konstruktionsplan selbst zugrundelegt, nicht erlauben, das Problemfeld der Klassiker selbst darzustellen. Alexander entwickelt die Gedanken der Klassiker in einem Themenfeld, das $\mathrm{zu}$ arm ist, um die Problemlage dieser Klassiker zu verstehen.

Folgenlos bliebe dies nur dann, wenn es sich hier um philosophische Hintergründe handelte, die der soziologischen Theoriebildung äußerlich sind. Vielleicht denkt Alexander so. Festzuhalten wäre aber auch in diesem Fall, daß Alexanders Konstruktion gegenüber der philosophischen Problemlage bei den Klassikern der Soziologie unsensibel und ungerechtfertigt ist. Dies gilt übrigens auch für die heutige philosophische Problemlage. Um dies nur an einem Beispiel klarzumachen: im Gefolge Wittgensteins hat sich wie zuvor schon unter dem Einfluß des Pragmatismus eine interne Verknüpfung von Handlungs- und Sprachtheorie ergeben, die von keinem zeitgenössischen Versuch einer soziologischen Handlungstheorie einfach ignoriert werden kann. Alexanders Konstruktion bleibt aber ebenso wie die von Parsons hiervon völlig 
unberührt, Vergessen wir nicht, worauf besonders Charles Taylor überzeugend hingewiesen hat, daß schon zu Kants Lebzeiten die Reflexion auf die Sprache und das Problem der sprachlichen Bedeutung - bei Herder und Humboldt - der Antinomie von Kantianismus und Utilitarismus eine neue Denkmöglichkeit hinzufügte, welche insbesondere in der deutschen Geistesgeschichte einerseits auf dem Wege zu Marx, andererseits auf dem Wege zu Hermeneutik und philosophischer Anthropologie eine reiche Wirkung entfaltete (Taylor 1986).

An einer zweiten Thematik läßt sich allerdings zeigen, daß Alexanders Konstruktion und die mangelnde philosophische Reflektiertheit ihrer Prinzipien keineswegs folgenlos bleiben. In Parsons' Konzeption war die Zentralstellung des Problems einer Handlungstheorie, die alternativ zur Theorie des rationalen Handelns angelegt war, und des Problems der sozialen Ordnung, welches eben mit Mitteln einer Theorie rationalen Handelns nicht lösbar sei, schnell gerechtfertigt. In Alexanders Konstruktion fehlt zunächst ein hinreichendes Argument für seine mit großem Nachdruck vorgetragene Behauptung, die Probleme von Handlung und Ordnung seien die Schlüsselprobleme jeder soziologischen Theorie. Sein jüngstes Buch liefert hier am Rande ein Argument nach; demnach stellt der Versuch zur Integration individueller Freiheit und sozialer Ordnung ein typisch modernes, typisch westliches Kulturproblem dar. Dies klingt plausibel und ist auch durchaus mit Parsons' Ambitionen verträglich; es führt nur insofern in Schwierigkeiten, als dieses Problem damit in seiner kulturellen Bedeutung ja relativiert wird und nicht mehr selbstverständlich als universelles, rein theoretisches Zentralproblem aufgefaßt werden kann. Zudem wird damit erst recht die Notwendigkeit deutlich, in einem weiteren Horizont der Reflexion über Freiheit, Individualismus und soziale Ordnung zu denken als in dem des Konflikts zwischen autonomer Moralität und egoistischem Interessenkalkül.

Auch das genannte Argument tritt aber nur am Rande auf. Alexander bietet zwar ausführliche Darlegungen über die Einbettung der Wissenschaft in eine „metaphysische“ und eine „empirische“ Umwelt und die verschiedenen „Komponenten" des von ihm als "scientific continuum" bezeichneten Bereichs zwischen diesen Polen. Zweck dieser Darlegungen ist es, verschiedene Ebenen der wissenschaftlichen Argumentation so zu unterscheiden, daß sowohl die irrtümliche Plazierung von Argumenten verhindert werden wie insbeson- dere eine Ebene allgemeinster Präsuppositionen identifiziert werden kann. Damit will er die explizite Diskussion von Präsuppositionen möglich machen. Erstaunlicherweise bezieht er dies aber nicht auf seine eigenen Präsuppositionen, die ja eben in seinem Konstruktionsplan und den allgemeinsten Schemata seiner Argumentation stecken. Die Behauptung, Handlung und Ordnung seien die Zentralprobleme, findet ebensowenig ausführliche Begründung wie die Unterscheidung von Haupttypen der Handlungstheorie (rational-instrumentell versus nichtrational-normativ) oder der Theorie sozialer Ordnung (individualistisch-emergent versus kollektivistisch-sui generis). Nichts wäre doch wesentlicher gewesen für die Überzeugungskraft von Alexanders Argumentation, als eben diese zentralen Unterscheidungen nicht einfach $z u$ setzen und dann schematisch anzuwenden, sondern gegenüber der heutigen und der für die Begründung der Soziologie konstitutiven Problemlage erst einmal zu begründen. Treffen diese Unterscheidungen denn überhaupt die Problemlage im Werk der wichtigsten Theoretiker oder stellen sie ein artifizielles Schema dar, einen Leisten, über den alles ohne Rücksicht auf Verluste nur geschlagen wird?

Mit dieser merkwürdigen Lücke in der Argumentation hängt unmittelbar ein weiteres Problem zusammen. Alexander legt wie Parsons großen Nachdruck auf den analytischen Status theoretischer Abstraktionen; davon war bei der Kennzeichnung der Theorien rationalen Handelns kurz die Rede. Wir erfahren aber nichts darüber, wie nun eigentlich auf dieser analytischen Ebene argumentiert werden kann. Es muß doch rationale Gründe dafür geben, in welcher Richtung ein analytisch aufgefaßter metatheoretischer Bezugsrahmen eigentlich entwickelt werden darf und in welcher nicht. Wenn die Betonung des analytischen Charakters einer vorgelegten Theorie nur zur Abwehr von Einwänden dient, ist die Gefahr, daß sich eine spezifische Metatheorie schlicht gegen Kritik immunisiert. Wenn ich richtig sehe, gibt es für eine analytische metatheoretische Argumentation nur zwei Weisen der Begründung. Es kann zum einen pragmatische Gründe dafür geben, eine bestimmte Abstraktion vorzunehmen - etwa die des homo oeconomicus ohne damit irgendeinen Realitätsstatus für diese Abstraktion zu behaupten. Es kann zum anderen ontologische Gründe geben, daß eben eine spezifische Abstraktion dem Wesen des Menschen oder dem Menschen des bürgerlichen Zeitalters o. ä. am besten entspricht. Aufgaben und Arbeitsteilung der Wissenschaften einerseits, anthropologische Annahmen oder historische Idealtypen anderer- 
seits berechtigen zu spezifischen analytischen $\mathrm{Ab}$ straktionen. Wenn dies zutrifft, dann muB sich eine soziologische Handlungstheorie entweder mit dem pragmatischen Nutzen ihrer spezifischen analytischen Abstraktion begründen. Parsons schwebte mit seiner These von der Soziologie als der Spezialdisziplin für normative Integration wohl Derartiges vor; Alexander übernimmt diese Aufgabenbestimmung aber ausdrücklich nicht. Oder aber anthropologische Annahmen über die Charakteristika des menschlichen Handelns - entweder im Vergleich zu tierischem Verhalten oder über historisch dominante Handlungstypen - erlauben die Begründung des gewählten Kategorienapparats. Auch davon finden wir bei Alexander nichts. Damit aber verlieren seine begrifflichen Grundunterscheidungen ihren Boden. Was bei Parsons noch aus dem Bezug zur ökonomischen Methodenkontroverse gut nachvollziehbar war, erhält sich bei Alexander bloß als Reminiszenz. Er geht nicht den Weg, die konkurrierenden Abstraktionen (rational/nichtrational) neu zu durchdenken, sondern proklamiert lediglich die Aufgabe, beide Typen von Handeln gleichzeitig zu berücksichtigen. Die Aufgabe der Entwicklung eines befriedigenden handlungstheoretischen Begriffsapparats ist damit aber noch nicht ansatzweise gelöst. Thomas Burger hat darauf hingewiesen, daß ein hinter Abstraktionen zurückgehender Konzeptualisierungsversuch etwas prinzipiell anderes ist als die gleichzeitige empirische Berücksichtigung verschiedener Einflußdimensionen (Burger 1986: 278). Dies scheint mir ein entscheidendes Argument zu sein zur Beurteilung von Alexanders Anspruch, eine multidimensionale und synthetische Theorie vorgelegt zu haben. In der Kritik an anderen Theorien kann Alexander zwar von seinem Standort aus zeigen, daß die Vernachlässigung einer Dimension schädliche Folgen haben kann und tatsächlich häufig hat. Er kann aber bereits nicht ausschließen, daß die Vernachlässigung einer Dimension (der "normativen" oder der "materiellen") für die Zwecke einer bestimmten empirischen Erklärung folgenlos bleibt, da diese tatsächlich hier praktisch vernachlässigt werden kann. Wenn er aus seinem Anspruch der Multidimensionalität die positive Behauptung ableiten möchte, alle Dimensionen müßten stets empirisch gleich wichtig sein, wäre dies bereits eine falsche Konkretisierung der analytischen Multidimensionalität. Darüber hinaus ist aber jede positive empirische Folgerung aus dieser Metatheorie verboten. Was sich als Synthese versteht, ist deshalb praktisch nur ein leeres ,sowohl-als auch“.
Noch in einer dritten Hinsicht erweist sich Alexanders theoretische Konstruktion, die doch die Handlungstheorie für so zentral erklärt, als gerade handlungstheoretisch ungenügend, und zwar in Hinsicht auf den Status allgemeiner Theorie und das „scientific continuum“. Viele Kritiker haben gerade diesen Teil von Alexanders Unternehmung und überhaupt den ersten Band seiner „Theoretical Logic“ scharf negativ beurteilt. Mißt man ihn am wissenschaftstheoretischen Schrifttum, auf das Alexander sich hier ja stützt, dann schneidet dieser Text sicher nicht gut ab; legt man dagegen das Reflexionsniveau praktizierender Sozialforscher zugrunde, dann liegt hier durchaus ein nützlicher Beitrag zur wissenschaftstheoretischen Reflexion der Soziologie vor. Verschiedene Kritiker haben behauptet, daß Alexander die relative Autonomie grundlegender theoretischer Annahmen gegenüber Empirie und Erfahrung stärker hervorhebe als die umgekehrte Relation: daß auch Tatsachen zwar theoriegeleitet formuliert, durchaus aber in konkurrierenden Theorien gemeinsam anerkannt werden könnten. Damit aber laufe Alexander Gefahr, einer Art theoretizistischem Determinismus zu verfallen. So richtig dies ist, scheint mir doch der springende Punkt an Alexanders Konstruktion des „scientific continuum“ damit noch nicht getroffen. Alexander konstruiert die Situation der Erkenntnis zwischen metaphysischer WelterschlieBung und alltäglicher Erfahrung nicht selbst als Handlungssituation! Wie schon Parsons verfehlt auch er den pragmatistischen Grundgedanken einer Situierung des Erkennens in problematischen Handlungssituationen. Sein Handlungsbegriff weist keinen internen Bezug zu einem Begriff des Wissens auf. Damit aber wird das Verhältnis von WelterschlieBung und praktischer Erfahrung zu einem der Über- oder Unterordnung und nicht wie etwa bei Toulmin (1983) - zu einer wechselseitigen Verschränkung. Alexander konzipiert das gesellschaftstheoretische Denken nicht als praktische Selbstreflexion von Akteuren und verfehlt damit auch eine neue Art der Begründung für die Zentralstellung der Handlungskategorie. Wenn die Erkenntnis notwendig ans Handeln gebunden ist, muß doch jede wissenschaftliche Theorie letzten Endes auf ihren Ort im menschlichen Handeln reflektieren.

Ein Zwischenergebnis zeichnet sich damit ab. Zwar kommt Alexander das Verdienst zu, die Bedeutung allgemeiner soziologischer Theorie und insbesondere der Handlungs- und Ordnungstheorie zu verfechten. Doch hat er weder für die historische Analyse der Entstehung der soziologischen Handlungstheorie noch für deren interne Ausar- 
beitung noch für die Reflexion auf den wissenschaftlichen Status dieser Theorie entscheidend Neues beigetragen. Die Aufmerksamkeit sollte deshalb jetzt auf jenes Gebiet umgelenkt werden, auf das sich offensichtlich auch der größte Teil von Alexanders Anstrengungen richtete: die KlassikerInterpretationen. Hier kann an der grundsätzlichen Qualität der Arbeit eigentlich kein Zweifel sein. Die Argumentation ist immer klar und fair, der Überblick über Primär- und Sekundärliteratur beeindruckend. Die Verknüpfung von theoretischer Argumentation und interpretatorischer Auseinandersetzung ist sicher notwendig. Die rein historische Beschäftigung mit den soziologischen Klassikern wäre ja für die Soziologie unmotiviert; die rein theoretische Argumentation würde ohne den Focus des Bezugs zu den Klassikern an ihrer Überkomplexität verenden. Fraglich ist hier also nicht diese Verknüpfung selbst, sondern lediglich ihr Gelingen. Fraglich ist allgemein, ob der verwendete metatheoretische Rahmen hermeneutisch geschmeidig genug ist, um nicht nur einzelne Problemfelder $\mathrm{zu}$ identifizieren, sondern die selbstgestellten Aufgaben der interpretierten Autoren und die Ausrichtung des eigenen Fragens ,wirkungsgeschichtlich" (Gadamer) zu vermitteln. Davon hängt es ab, ob das Werk eines Autors als Haufen widersprüchlicher Aussagen zu den Fragen des Interpreten erscheint oder ob es gelingt, im Horizont des eigenen Fragens die Fragen und die Antworten eines Autors zu verstehen, auch ein mögliches Schwanken oder innere Widersprüche zu erfassen, vielleicht sogar die Folgeprobleme von Lösungsversuchen zu analysieren und auf diesem Wege tatsächlich einen Autor besser zu verstehen als er sich selbst verstehen konnte. Dieses hermeneutische Ziel hatte Alexander ja in der Wahl des Schleiermacher-Mottos im Weber-Band sich - mit Recht - zu eigen gemacht. Fragen des Einflusses oder der Periodisierung und Datierung erhalten dadurch ihre über die philologische Empirie hinausreichende theoretische Dignität. Leider verfährt Alexander damit im einzelnen manchmal recht sorglos. Immer wieder behauptet er in irritierender Weise Entwicklungen, die er philologisch nicht beweisen kann und als rein metatheoretische Frage prompt von der hermeneutischen Empirie wieder isoliert. Von theoretischem Interesse sind aber ebenfalls nicht mögliche Kunstfehler Alexanders, sondern lediglich solche interpretatorischen Probleme, die sich auf Probleme seines metatheoretischen Rahmens zurückführen lassen. Fast ausschließlich diesen gilt deshalb die Aufmerksamkeit in den folgenden Abschnitten.
Die Marx-Interpretation Alexanders verknüpft die bei Parsons nicht in ihrem Verhältnis zueinander geklärten Charakterisierungen. Marx entstamme zwar einem normativen Idealismus und seine frühesten Schriften seien ohne den Konflikt zwischen einer universalistischen Moral und den vorfindbaren institutionalisierten Normen unverständlich. Der Bruch in seiner Werkentwicklung aber bestehe dann in der (vermeintlichen) Erkenntnis von der Machtlosigkeit moralischer Kritik und in der Auffassung, für das Zeitalter der Entfremdung bzw. für den Kapitalismus sei nicht von einer Anthropologie des freien, sich ausdrückenden und vergegenständlichenden Menschen auszugehen, sondern von dem in der klassischen politischen Ökonomie gefaßten Modell utilitaristisch-egoistischen Handelns miteinander konkurrierender Einzelsubjekte. Die Debatte um einen Bruch in der Entwicklung des Marxschen Werkes wird damit um eine weitere Variante bereichert: Alexander datiert den Bruch so früh wie keiner zuvor, nämlich auf die Jahre 1842/43, sieht damit bereits die „Pariser Manuskripte" nicht als Werk des frühen Marx, sondern schon als Teil einer lediglich noch ambivalenten, 1845 abgeschlossenen Übergangsphase zum reinen Utilitarismus. Der Bruch, wie er ihn sieht, fällt damit eigentlich mit der Rezeption der politischen Ökonomie durch Marx zusammen. Theoretisch heißt dies, daß Marx den Utilitarismus nicht etwa überwindet, sondern ihn im Gegenteil voll akzeptiert, lediglich aber geschichtsphilosophisch in seiner Geltung relativiert. Diese These, welche den Kern von Alexanders Marx-Deutung ausmacht, wird insofern spezifiziert, als nur von einer Aufnahme der utilitaristischen Handlungs-, nicht aber der Ordnungstheorie gesprochen wird. Auf ordnungstheoretischer Ebene trete Marx mit der Betonung des Klassenscharakters der existenten Ordnungen den utilitaristischen Theorien gerade entgegen. In dieser theoretischen Grundstruktur sieht Alexander die systematische Unfähigkeit des Marxismus verankert, über eine instrumentalistische Staatstheorie und eine funktionalistische Reduktion von Recht, Moral und Ideen hinauszugehen.

Es ist außer Zweifel, daß Alexander damit ein Problem berührt, das auch bei anderen Autoren und auch innerhalb der marxistischen Diskussion aufgeworfen wurde. Die Frage lautet, wie denn eine tatsächlich auf utilitaristische Weise verfaBte Kritik der politischen Ökonomie mit der Revolutionstheorie oder allgemeiner politischen Soziologie des Marxismus verknüpft werden könne, wenn nicht von vornherein die handlungstheoretischen 
Grundlagen beider Theoriebestandteile aufeinander bezogen sind. Alexander verficht die These vom utilitaristischen Charakter der systematischen Theorie Marxens in besonderer Radikalität; dies stellt eine heilsame Nötigung zur Klärung dar, da er damit beliebte Auswege verbaut: Er akzeptiert nicht die Behauptung von der Überlegenheit von Marx' faktischer Forschung über seine methodologischen Selbstdeutungen, da der UtilitarismusVorwurf gerade für die faktische Forschung gelte; er lehnt Verweise auf Marx' allgemeines Weltbild, aber auch auf seine politische Publizistik und sogar die historiographischen Arbeiten ab, da diese zwar Ausdruck des stillschweigenden Wissens von Marx, nicht aber der systematischen Folgen seines Begriffssystems seien; schließlich weist er die Betonung einzelner Marxscher Verweise auf kulturelle Elemente als Argument gegen seine Interpretation zurück und sieht in ihnen eher einen Beleg für deren Richtigkeit, insofern sich in jeder Theorie systematisch Ausgeschlossenes in der Gestalt von Residualkategorien zu melden pflegt. Dies alles ist äußerst ernst zu nehmen. Dennoch bleiben zwei Einwände, die geeignet sind, Alexanders MarxDeutung teilweise zu erschüttern.

Der eine Einwand zielt auf die Spezifik von Marx' Kritik der politischen Ökonomie gegenüber der klassischen politischen Ökonomie im allgemeinen. Alexanders Rekonstruktion läßt eine Differenz zum Utilitarismus nur auf ordnungstheoretischer Ebene gelten. Dem steht aber doch entgegen, daß Marx' spezifische Version von Arbeitswertlehre mit all ihren Folgen von der Mehrwerttheorie bis zum Gesetz vom tendenziellen Fall der Profitrate unverständlich bleibt, wenn nicht ihre Fundierung in dem nicht-utilitaristischen, sondern ,expressivistischen“ (Charles Taylor) Begriff der Arbeit gesehen wird. Wenn dies zutrifft, ist ein ganz anderer Strang von Handlungstheorie noch im Werk des späten Marx von entscheidender Bedeutung und läßt sich die Bedeutung der Praxis-Anthropologie nicht auf einen bloß normativen Hintergrund einer kategorial anders verfaßten Theorie reduzieren. Damit soll aber nicht der Behauptung widersprochen werden, da $B$ Marx diesen fundierenden Begriff selbst nicht ausgearbeitet und insbesondere nicht in seiner Bedeutung für die Analyse des Bewußtseins der Lohnarbeiter und ihres politischen Handelns ernstgenommen hat. Auf diesen Bereich zielt der zweite Einwand. Alexanders Argumentation setzt voraus, daß allein die Kritik der politischen Ökonomie als wissenschaftlicher Teil des Marxschen Werkes betrachtet und daß diese zugleich mit dem Anspruch einer Gesellschafts- theorie belastet wird. In dieser Hinsicht, in der Gleichsetzung des „Kapital“" mit Marx' Gesellschaftstheorie, ist Alexander so entschieden wie die trockensten „Kapital“-Exegeten des bundesdeutschen Seminar-Marxismus der siebziger Jahre es waren. Dies ist aber nicht selbstverständlich und wirft die Frage nach dem Status der Kritik der politischen Ökonomie in einer Gesellschaftstheorie auf. So richtig es ist, daß die Marxsche Kritik ebensowenig übrigens wie die klassische politische Ökonomie - sich nicht mit einem disziplinär-ökonomischen Status bescheidet, so falsch wäre es, sie einfach zur umfassenden Soziologie zu erklären. Nur dann wäre für eine Theorie des Staats, des Rechts, der Familie oder der Kunst nichts anderes als der Status quasi-funktionalistischer „Ableitungen“ möglich. Sieht man dagegen im „Kapital“ die Herausarbeitung von Entwicklungstendenzen auf hoher Abstraktionsebene, dann ist über das Verhältnis dieser zu anderen hiermit noch gar nicht entschieden. Alexanders Deutung projiziert die Problemlage der Klassiker der Soziologie in der Generation von Max Weber, Emile Durkheim und Vilfredo Pareto auf Marx zurück, in dessen Zeit der verwissenschaftlichen Ökonomie lediglich eine politische Philosophie zur Seite stand: bei Marx wie bei den von Marx kritisierten Autoren.

Die Traditionen dieser politischen Philosophie aber lassen sich nicht aus dem Konflikt von Utilitarismus und Normativismus heraus verstehen. Sie gehen auf die praktische Philosophie des Aristoteles zurück und erfahren in der Reaktion auf utilitaristische und materialistische Tendenzen der Aufklärung mannigfache Transformationen. Herders und Humboldts Ausdrucksanthropologie, Fichtes Interpersonalitätskonzeption, Hegels Versuch einer Aufladung der praktischen Philosophie mit dem Freiheitspathos der transzendentalphilosophischen Fassung des Naturrechts, Feuerbachs anthropologischer Materialismus - all dies sind Formen des Denkens, die sich an Kant vorbei oder über Kant hinaus entwickelten. Schon bei Parsons war im Vorspann seiner Weber-Interpretation das Bild vom deutschen Idealismus ganz unzureichend geblieben. Fälschlich projizierte er die Tendenz des Historismus, soziale Phänomene als Emanation oder Ausdruck kultureller Sinzusammenhänge zu deuten, auf den ganzen deutschen Idealismus; er verkannte dadurch insbesondere die $\mathrm{Be}$ deutung des Ausdrucksmodells des Handelns, weil er zwar zu Recht die Deutung von Handlungen als Ausdruck eines „objektiven Geistes“ ablehnte, das Potential der Deutung von Handlungen als Ausdruck des Handelnden aber gar nicht auslotete. 
Wenn Alexander zwar anders als Parsons Marx ausführlich behandelt, die ungenügende Berücksichtigung des philosophischen Weges zu Marx aber perpetuiert, dann ist dies nur ein kleiner Fortschritt. Wenn Hegels Einfluß auf Marx geringzuschätzen sei - wie Alexander behauptet - dann bleibt immer noch die Frage, ob dann nicht Hegels Theorie selbst sich Alexanders Schema entzieht. Es ist hier nicht der Ort, um die Geschichte des deutschen Idealismus unter handlungstheoretischem Gesichtspunkt zu entwickeln. Wichtig ist allerdings die Erkenntnis, daß der Schlüsselbegriff des „Geistes“ nicht als „normative Dimension“ rekonstruiert werden kann, sondern vielmehr eine spezifische Form der Kennzeichnung der Kreativität der Tätigkeit darstellt. In Marx' Werk selbst finden sich mindestens zwei Handlungsmodelle, die sich weder als utilitaristisch noch als normativistisch kennzeichnen lassen (Honneth/Joas 1987). Vom expressivistischen Arbeitsbegriff war bereits die Rede; besonders in den Frühschriften macht Marx klar, daß für ihn menschliche Arbeit nicht bloß einen Prozeß der produktiven Verausgabung von Leistungen darstellt, sondern zugleich als ein Ausdrucksgeschehen zu betrachten ist, durch das der Mensch sich selbst zu verwirklichen versucht. Der Kapitalismus verfällt gerade deshalb der Marxschen Kritik, weil er die Möglichkeit der Selbstverwirklichung, d.h. das Wiedererkennen der arbeitenden Subjekte in ihren eigenen Produkten, strukturell erschwert oder verunmöglicht. Neben diesem expressivistischen Modell der Arbeit, auf dessen Probleme hier nicht einzugehen ist, findet sich insbesondere in den historischen Arbeiten ein Modell kollektiven Handelns, das eben nicht nur die normativen Dimensionen des Klassenkampfes miteinbezieht, sondern die kreativnormsetzenden Leistungen sozialer Gruppen herausarbeitet. Der Klassenkampf ist bei Marx nicht nach dem Schema nutzenorientierten Interesssenhandelns zu verstehen, da Marx gerade Selbstverwirklichung und Selbstbestimmung zum Interesse des Proletariats erklärt. Keineswegs soll damit behauptet werden, daß Marx' Lösungsversuche unproblematisch seien. Die Synthese von Ausdrucksanthropologie und Utilitarismus ist in beiden Fällen: dem Begriff der Arbeit wie dem des Klassenkampfs, gleich labil. Zudem ist das Verhältnis von politökonomischer und klassenkampfzentrierter Geschichtsdeutung - wie vor allem Castoriadis (1984; vgl. auch Berki 1979) herausgearbeitet hat nie wirklich geklärt worden. Alexanders handlungstheoretische Schematik enthält aber überhaupt keine Möglichkeit, diese Probleme auch nur zu registrieren.
Das Grundmotiv der Durkheim-Deutung Alexanders ist die Suche nach einem Begriff sozialer Ordnung, der mit der Freiwilligkeit des Individuums vereinbar ist. Alexanders Ziel ist es, detaillierter als Parsons die windungsreiche Entfaltung der Problemlösung Durkheims, nämlich der Einsicht in die Sozialität des Akteurs, herauszuarbeiten. Die verschiedenen gedanklich erprobten Ansätze in den frühen Schriften werden ebenso wie die Schritte zur Konzeption ,emotionaler" und nicht „instrumentaler“ Interaktion im Umkreis der religionssoziologischen Beschäftigung Durkheims differenziert aufgewiesen. Im Unterschied zu Parsons sieht Alexander Durkheim nicht auf dem Wege vom Positivismus zum Idealismus, sondern von einer normativistischen Frühphase über eine materialistische Zwischenphase zum immer normativistischer oder idealistischer werdenden Spätwerk. Die krasseste Differenz zu Parsons' Interpretation ergibt sich dabei nicht aus der stärkeren Berücksichtigung der frühen Schriften Durkheims, sondern bei der zu Parsons gegenläufigen Deutung des „Arbeitsteilungs“-Buchs. Von Parsons bis Giddens gilt dieses Buch im allergrößten Teil der gesamten Durkheim-Literatur als klassische Kritik des Utilitarismus durch den Nachweis der nicht-kontraktuellen Voraussetzungen des Vertrages und der moralischen Grundlage auch der modernen Gesellschaft. Für Alexander stimmt dies gerade nicht und erwächst diese Fehldeutung aus einer isolierten Berücksichtigung des ersten Teils der „Arbeitsteilung“. Für ihn formuliert Durkheim eher eine Theorie der fortschreitenden Elimination dieser moralischen Basis und befindet sich damit eher auf dem Weg zurück zu Spencer oder dem als Utilitaristen gedeuteten Marx. Insbesondere Marx rücke hier nahe, insofern die Theorie der Solidaritätsformen direkt der geschichtsphilosophischen Relativierung des Utilitarismus bei Marx entspreche. Schon immanent zeigen sich in Alexanders Darlegung dieser kühnen These Schwierigkeiten, die nach den Erfahrungsregeln der Hermeneutik Anlaß zur Vorsicht geben: Alexander muß ein extremes Ausmaß innerer Inkonsistenz im „Arbeitsteilung"-Buch, konzeptionelle Schwankungen in der nachfolgenden Phase und förmliche Täuschungsmanöver im folgenden Buch über die ,Regeln der soziologischen Methode" unterstellen. Könnten diese Schwierigkeiten nicht ein Hinweis sein, daß auch bei Durkheim die Problemlage nicht einfach im Schema Normativität-Utilitarismus erfaßbar ist? Zusätzliche Nahrung erhält dieser Verdacht, wenn man die Chance wahrnimmt, jene Schrift Durkheims heranzuziehen, die Parsons noch nicht 
kennen konnte, da sie erst posthum veröffentlicht wurde, welche aber die Entwicklung Durkheims nach Abschluß der Religionstheorie beleuchtet: die Rede ist von den Vorlesungen über „Pragmatismus und Soziologie". Alexander geht auf diese überhaupt nicht ein. Sie können zeigen, wie wenig Durkheims halbherziger Versuch zur Reformulierung des Cartesianismus im Angesicht der pragmatistischen Kritik sich mit einer Kategorie wie ,soziologischer Idealismus" erfassen läßt (Durkheim 1987).

Wie schon im Falle von Marx kann auch bezüglich Durkheims eine alternative Interpretation nur angedeutet werden, um zu demonstrieren, wie notwendig ein weiterer metatheoretischer Rahmen als der von Alexander ins Spiel gebrachte wäre. Ich glaube nicht, daß wir Durkheims ,Arbeitsteilung“" Buch richtig erfassen, wenn wir in ihm entweder den positivistischen Nachweis der automatischen Entstehung moralischer Integration durch physische Interaktion (wie etwa Randall Collins) oder die blo $B$ abstrakte, quasi-utilitaristische Hoffnung sehen, instrumentell orientierte Handlungen mögen irgendwie ihre eigene Moralität beinhalten (wie Jeffrey Alexander). Mir scheint vielmehr, daß Durkheim in der "Arbeitsteilung" einen Gedanken verfechten wollte, zu dessen klarer Ausführung ihm die Denkmittel fehlten. Dies war der Gedanke einer Kooperationsmoral, d. h. einer Moralität, die aus den Erfahrungen und Erfordernissen gleichberechtigten Kooperierens durch die Selbstreflexion der Kooperierenden selbst erwachsen kann. Eine solche Kooperationsmoral wäre weder mit einer kausalen Folge des Kooperierens noch mit einer bloß frommen Begleitmusik zur Tauschwirtschaft identisch. Durkheim konnte dies aber nicht klarer formulieren, da ihm zum einen die entwicklungspsychologisch-sozialisationstheoretischen Mittel fehlten, um - wie Piaget (1973) in seinem frühen Meisterwerk über das moralische Urteil beim Kinde - die Genese einer Kooperationsmoral darzustellen. Deshalb klafft in seiner Konstruktion zwischen dem Nachweis der Häufigkeit yon Kooperationen und der behaupteten Entstehung einer Kooperationsmoral eine Lücke. Der andere Grund liegt in einer tiefen Zweideutigkeit in Durkheims Begriff der Arbeitsteilung, die bei Alexander unbemerkt bleibt. Durkheim unterscheidet nämlich nicht zwischen der antagonistischen Arbeitsteilung des Marktes und der nichtantagonistischen Arbeitsteilung organisierter Kooperation. Seine These von einer aus der Arbeitsteilung selbst erwachsenden Moral läßt sich sinnvoll nur für die aus egalitärer Kooperation resultie- rende Moral verteidigen. Die moderne Gesellschaft der kapitalistischen Industrialisierung, die Durkheim analysieren will, ist aber nicht von einer Ausbreitung solcher Kooperationsbeziehungen, sondern von der Freisetzung marktförmiger Prozesse und, wie Marx gesagt hätte, ihrer widersprüchlichen Einheit mit einer Ausdehnung hierarchischer innerbetrieblicher Kooperation gekennzeichnet. Durkheim muß deshalb in Widersprüche geraten, wenn er in dieser modernen Gesellschaft eine neue Moral erwartet, tatsächlich aber vornehmlich anomische Tendenzen konstatiert. Die Deutung dieser Phänomene als Übergangserscheinungen ist eine Ausflucht. Alexander faßt in seiner Interpretation nur einen möglichen Sinn von Arbeitsteilung: den marktförmiger Vernetzung individuell-nutzenorientierter Handlungen. Denkt man so, bleibt der Weg zur Kooperationsmoral natürlich völlig verschlossen und muß Durkheims Theorie dem klassich-utilitaristischen Modell der Marktvergesellschaftung angeähnelt werden. Durkheims Frage war dagegen die nach der Entstehung einer neuen Moral. Im weiteren Verlauf seiner Entwicklung löst er nicht die genannten Probleme, verändert aber seine Problemstellung. Immer stärker wird seine Überzeugung, daß eine solche Kooperationsmoral allein zu schwach ist, wenn sie aus der Kooperation selbst erwachsen soll; sie muß vielmehr selbst institutionalisiert werden. Dies führt ihn zu der Frage nach der Entstehung von Institutionen, und diese Frage beantwortet er mit der Theorie des Rituals und der kollektiven Efferveszenz. Ich schlage deshalb vor, seine Entwicklung als die der Frage nach der Entstehung und kreativen Erzeugung von Institutionen und Weltbildern zu deuten. Auch in ihrer reifsten Variante aber gelingt es Durkheim nicht, die Einsicht in die Bedeutung außeralltäglicher kollektiver Interaktion mit einer Theorie alltäglicher sozialer Interaktion zu verknüpfen. Alexander sieht sehr wohl, daß Durkheims Handlungsbegriff utilitaristische Reste behält und das Soziale bei ihm gerade vom Handeln abgetrennt wird. Durkheims Persönlichkeitsbegriff kombiniert ein substantialisiertes biologisches Individuum mit einem sozialen $\mathrm{Be}$ griff der Persönlichkeitsstruktur. Hier ist Meads Denken, das von der sozialen Formung der biologischen Antriebe ausgeht, viel radikaler. Wenn es also nur um den Nachweis des sozialen Charakters der Person ginge, wäre Durkheim gar nicht der entscheidende Autor. Durkheim ist aber gerade für jenen Punkt entscheidend, den Alexanders metatheoretischer Rahmen nur schwer zu bezeichnen erlaubt: die Erzeugung von Institutionen und 
Weltbildern in Prozessen des kollektiven Handelns.

Uberwogen bei der Auseinandersetzung mit Alexanders Interpretation von Marx und Durkheim die Einwände, so ändert sich dies bei der Behandlung von Weber und Parsons. Im Falle Webers enthält sich Alexander weitgehend aller philologischen Behauptungen über die Entwicklung des Autors. Er sieht eher eine permanente Spannung wirken, die verschiedenen Teilen des Werks sehr unterschiedlichen Charakter gibt. Die Spannung ist erneut die von Normativismus und Utilitarismus, aber im Falle Webers erweisen sich diese Bestimmungen als recht hilfreich. Alexander sieht Weber als mit dem Versuch beschäftigt, „Ideen“ und „Interessen" integriert zu erfassen. Diese Integration sei in Teilen des Werkes wie der Klassentheorie und der Stadtsoziologie vorbildlich gelungen. Andere Teile wie die vergleichenden Studien zur Wirtschaftsethik der Weltreligionen seien hier höchst ungleich ausgefallen. Dem negativen Fall der China-Studie wird so die Arbeit zum antiken Judentum als positive Alternative entgegengestellt. Entscheidend ist aber, daß es sich hier nicht um nebensächliche Mängel der Ausarbeitung handeln kann, da Weber in einem wesentlichen Teil seines Werks konsistent reduktiv verfahre: in seiner Analyse der Moderne selbst. Sei noch die Analyse von deren Entstehung "multidimensional", so ändere sich dies programmatisch bei ihrer Darstellung selbst. Ob Soziologie des Rechts oder der Schichtung, ob Soziologie der Bürokratie oder der Demokratie: überall setze sich die einseitige Betonung utilitaristischer Züge durch und führe zu empirischen Schwächen. Die mögliche Begründung hierfür, dies sei eben Ausdruck des Wesens dieser modernen Gesellschaft, lehnt Alexander zu Recht ab, da mit Webers Vorgehen ja metatheoretisch vorentschieden werde, was empirisch $\mathrm{zu}$ beweisen wäre. Auf weiten Strecken ähnelt hier Alexanders Argumentation der sicher noch raffinierteren Beweisführung von Jürgen Habermas im Weber-Kapitel seiner „Theorie des kommunikativen Handelns" (1981) und unterscheidet sich stark von Parsons' Weber-Bild. Ausgezeichnet arbeitet Alexander die innere Zweideutigkeit von Webers Begriff der Zweckrationalität und damit die Unklarheit der von diesem Begriff abhängigen Handlungstypologie heraus. Er sieht Webers Schwanken zwischen einer utilitaristischen Deutung der Zweckrationalität und einer pragmatistischen Version, in der auch die Ziele oder Zwecke auf die Situationsgegebenheiten hin reflektiert werden. Wenn diese Kategorie und Webers Handlungs- theorie aber zweideutig sind, dann hat dies Folgen für den Begriff der Rationalität, der für Webers geschichtliche Analysen so zentral ist. Stellt dann die Moderne das Zeitalter freigesetzten individuellen Nutzenhandelns oder einer gesteigerten, pragmatisch vernünftigen Handlungsautonomie dar? Was ist dann unter einem Prozeß der Rationalisierung eigentlich zu verstehen?

Ich halte diese Fragen, die Alexander aufwirft, für berechtigt und außerordentlich wichtig. Es könnte lediglich sein, daB zu ihrer Beantwortung Alexanders Kategorienschema erneut nicht ausreicht. Es enthält ja nur einen sozusagen kantischen Begriff moralischer Autonomie. Webers Weltbild war aber viel stärker von den kreativitätsorientierten, genieästhetischen Zügen der Nietzscheschen Persönlichkeitstheorie geformt (Hennis 1987). Ähnlich wie Alexander bei Marx den Begriff des Klassenkampfs seiner nicht-utilitaristischen Züge beraubte, erkennt er bei Weber die von Nietzsche stammenden Züge des Machtbegriffs nicht und deutet deshalb Webers inhaltlosen Machtbegriff (III, 92) in utilitaristischem Sinne um. Webers Prägung durch Nietzsche einerseits, die historische Schule der deutschen Nationalökonomie andererseits wird mit den Begriffen „soziologischer Idealismus" oder "Normativismus" sicher nicht getroffen. Es kann hier nicht verfolgt werden, wie sehr auch Webers Theorie der Moderne von diesem Hintergrund aus, von den Chancen für die machtvolle Entfaltung selbstverantwortlicher großer Individuen geprägt ist. Hier reibt sich erneut Alexanders Schema schmerzhaft am analysierten Gegenstand - aber doch mit spürbar geringeren Reibungsverlusten als bei Marx und Durkheim.

Die Auseinandersetzung mit Parsons im vierten Band von Alexanders Werk ist sicher der gelungenste Teil der ganzen Unternehmung. Hier hat Alexander in der Durchdringung von Parsons' Schriften und der Berücksichtigung der kritischen Literatur zu Parsons eine enorme Leistung vorgelegt. Als selbstkritische Reform einer ParsonsSchule wäre dieser Band - isoliert genommen wohl tatsächlich so positiv aufgenommen worden, wie es die Ankündigungen für das Gesamtwerk versprachen. Ohne den geringsten Zweifel an seiner Bewunderung für Parsons' Lebenswerk zu lassen, verfährt Alexander unnachsichtig mit Parsons, wo dieser die selbstgesetzten Ziele nicht erreicht. Dies gilt auf methodologischer und substantieller Ebene, für die Sozialisationstheorie ebenso wie für die politische Bescheidung in den USA der Nachkriegszeit. 
Die wohl wichtigste theoretische Korrektur besteht darin, daß Alexander das berühmte AGILSchema der Austauschbeziehungen zwischen funktionalen Teilsystemen von seiner funktionalistischen Lesart befreit. Für ihn ist dieses Modell als funktionalistisches Gesellschaftsmodell nicht zu halten; es formuliert lediglich auf metatheoretischer Ebene eine Systematik analytischer Abstraktionen. Dies soll hier nicht näher referiert werden. Fragen wir besser sofort, ob sogar hier Probleme der Deutung aus Alexanders Ansatz resultieren. Ich sehe vor allem ein solches Problem. Parsons' Theorie enthielt kein Mittel, um die historisch kontingente kreative Erzeugung von Institutionen und Weltbildern zu erfassen. Alle Prozesse, die seine Theorie erfaßt, betreffen die Internalisierung oder Institutionalisierung prä-konstituierter Werte oder die Generalisierung, Modifikation oder Spezifikation dieser Werte. An dieser Schwäche ändert auch Alexanders Kritik nichts. Was bei Durkheims Institutionentheorie, Marx Begriff des Klassenkampfs und der Arbeit und Webers Begriff der Macht ausgespart bleibt oder unter die Normativität subsumiert wurde: die Kreativität des individuellen oder kollektiven Handelns, findet bei Parsons und in seiner immanenten Kritik keinen Anhaltspunkt ${ }^{3}$. Dies ist freilich selbst kein immanentes Argument.

Jeffrey Alexander hat seiner Auseinandersetzung mit den Klassikern des Faches Soziologie mittlerweile eine Darstellung der Soziologiegeschichte nach 1945 hinzugefügt. Wenn der Duktus des vierbändigen Werks oft schwerfällig war, die Argumentation mit zahllosen Zitaten und umfassenden Verweisen auf Sekundärliteratur belastet, dann kommt dieses Buch mit um so leichterem Gepäck daher. Es entstammt Vorlesungen und behält diese Form bei, ist ungleich lesbarer und spannender. Nach einer vorzüglichen Darstellung von Parsons'

\footnotetext{
${ }^{3}$ In einem mit Paul Colomy verfaßten Aufsatz (Alexander 1988: 193-221) geht Alexander in dieser Hinsicht einen Schritt weiter. Er versucht hier eine Synthese von revidierter funktionalistischer Differenzierungstheorie und einer symbolisch-interaktionistischen Theorie des kollektiven Handelns. Auch seine handlungstheoretische Begrifflichkeit wird dort in Richtung auf „inventive" Komponenten des Handelns geöffnet. Eine Auseinandersetzung mit diesen Anzeichen einer Sprengung der in der „Theoretical Logic" vorgelegten Konzeption unterbleibt hier; sie müßte die Problematik des beibehaltenen Primats der Differenzierungstheorie nachweisen.
}

Werk und der notwendigen Korrekturen daran gewissermaßen einer Populärversion des vierten Bandes der "Theoretical Logic“ - stellt Alexander die Nachkriegssoziologie nach einem bestimmten einfachen Plan dar. Er betrachtet die verschiedenen Schulen, die seit den sechziger Jahren miteinander konfligieren und die Hegemonie von Parsons zerstörten, ohne daß eine von ihnen eine neue Hegemonie aufgerichtet hätte, als jeweils einseitige Betonung eines Moments, das in Parsons' Werk zu kurz gekommen sei, von dieser Schule aber nun in einer Ausschließlichkeit behandelt werde, die eine „synthetische" Theorie unmöglich macht. In dieser Sicht der Dinge wird Alexander dadurch beflügelt, daß er nur eine kleine Anzahl von logisch möglichen Kombinationen metatheoretischer Elemente überhaupt für denkbar hält und zugleich glaubt, diese im Schema der zwei Handlungs- und Ordnungstypen, das wir aus der „Theoretical Logic" kennen, identifiziert zu haben. So kann er Konflikttheorie (Rex), Austauschtheorie (Homans), Symbolischen Interaktionismus (Blumer, Goffman), Ethnomethodologie (Garfinkel), Kultursoziologie (Geertz) und Kritische Theorie (Marcuse) jeweils leicht einordnen. Die Konflikttheorie erweist sich dann als rationalistische Handlungs- und kollektivistische Ordnungstheorie; die Austauschtheorie kombiniert dagegen rationalistische Handlungs- und individualistische Ordnungstheorie; der Symbolische Interaktionismus dagegen sei eine Kombination von individualistischer Ordnungstheorie mit einer nicht-rationalen, normativistischen Handlungskonzeption. Und so weiter.

Erneut verwendet Alexander wenig Mühe darauf, das verwendete Schema selbst zu rechtfertigen. Erneut ist recht unklar, inwiefern er diese Schulen in ihrer Differenz zu Parsons betrachten will oder inwiefern er behauptet, für die Entwicklung dieser Theorien sei die Auseinandersetzung mit Parsons, die Abnabelung von einer Muttertheorie, faktisch konstitutiv. Wir müssen deshalb erneut selbst fragen, wie überzeugend die Deutungen mit Alexanders Schema sind.

Der überwältigendste Eindruck, wenn diese Überprüfung beginnt, ergibt sich nicht aus dem einschränkenden Charakter des Schemas, sondern der berücksichtigten Formen von Theorie. Alexanders Darstellung der Soziologieentwicklung nach 1945 ist in schamloser Weise amerikazentrisch. Wir hören buchstäblich kein Wort über soziologische Theoretiker oder fachfremde, für die heutigen Diskussionen aber zentrale Autoren wie die 
folgenden: Pierre Bourdieu, Cornelius Castoriadis, Michel Foucault, Alain Touraine aus Frankreich; Norbert Elias, Arnold Gehlen, Niklas Luhmann, Helmuth Plessner aus der Bundesrepublik Deutschland. Anthony Giddens' Theorie wird auf einer halben Seite behandelt; ebenso Jürgen Habermas. Ich denke, es wäre ehrlicher gewesen, von einer Geschichte der amerikanischen soziologischen Theorie bis etwa $1970 \mathrm{zu}$ sprechen. Weder die heutige amerikanische noch die europäische Theoriediskussion werden auch nur annähernd angemessen erfaßt. Die eigentlich spannende Frage nach dem Bild dieser Autoren in Alexanders Schema läßt sich deshalb gar nicht stellen.

Betrachtet man die behandelten Autoren und Theorien, dann wird schnell sichtbar, daß auch hier die Fassungskraft von Alexanders Deutungsschema am größten ist, wenn es sich tatsächlich um „abtrünnige“" Parsons-Schüler (wie Garfinkel oder Geertz) handelt oder sich die Theorien wirklich in Auseinandersetzung mit Parsons entwickelten. Wo Alexander versucht, von diesem Problemfeld unabhängige Theorien darzustellen, entstehen teilweise Karikaturen. Die Darstellungen von Peirce, Husserl und Merleau-Ponty etwa sind völlig unzuverlässig. Die Einschränkung der Kritischen Theorie auf Marcuse ist nicht zu verteidigen. Am gelungensten ist hier noch die Behandlung Meads. Die Auseinandersetzungen mit Rex, Homans, Blumer, Garfinkel und Geertz sind dagegen lesenswert.

Zurück bleibt der Eindruck eines außerordentlich belesenen und argumentationsfähigen Autors, der in einer gewiß nicht theoriefreundlichen wissenschaftlichen Umwelt zu Überlegungen über die Probleme des Handelns und der sozialen Ordnung vorstößt, ohne den Mut zu haben, sich auf den geistesgeschichtlichen Reichtum und die reale Vielfalt des heutigen Denkens tatsächlich einzulassen. Die Öffnung zur Theorie wird gleich mit einer schematischen Beschränkung theoretischer Möglichkeiten wieder zurückgenommen. Wo sich ungelöste Probleme in der Erfassung des menschlichen Handelns zeigen, werden diese oft schematisch zugedeckt. In dieser Form kann sich der Neoparsonianismus oder Neofunktionalismus zwar als weitere Stimme im Konzert soziologischer Schulen vernehmbar machen. Um zum allgemein akzeptierten theoretischen Gerüst des Faches zu werden, müßte er aber erst seine Kapazität zum Ernstnehmen der heute wirklich konkurrierenden Positionen erhöhen. Von einer Konkurrenzfähigkeit gegenüber den Syntheseversuchen von Habermas und Giddens kann sonst keine Rede sein.

\section{Literatur}

Alexander, J., 1982/83: Theoretical Logic in Sociology. 4 Bände. London: Routledge, (Band 1: Positivism, Presuppositions, and Current Controversies; Band 2: The Antinomies of Classical Thought: Marx and Durkheim; Band 3: The Classical Attempt at Theoretical Synthesis: Max Weber; Band 4: The Modern Reconstruction of Classical Thought: Talcott Parsons).

Alexander, J., 1984: The Parsons Revival in Germany. Sociological Theory 2: 394-412.

Alexander, J., 1985: Introduction. S. 7-18 in J. Alexander (Hrsg.), Neofunctionalism. London: Sage.

Alexander, J., 1986: Habermas' neue Kritische Theorie: Anspruch und Probleme. S. 73-109 in A. Honneth/H. Joas (Hrsg.), Kommunikatives Handeln. Frankfurt: Suhrkamp.

Alexander, J., 1987a: The Centrality of the Classics. S. 11-57 in A. Giddens/J. Turner (Hrsg.), Social Theory Today. Cambridge: Polity Press.

Alexander, J., 1987b: Sociological Theory since 1945. London: Hutchinson.

Alexander, J., 1988: Action and Its Environments. New York: Columbia University Press.

Berki, R. N., 1979: On the Nature and Origins of Marx's Concept of Labour. Political Theory 7: 35-56.

Burger, Th., 1986: Multidimensional Problems. Sociological Quarterly 27: 273-292.

Camic, Ch., 1987: The Making of a Method: A Historical Reinterpretation of the Early Parsons. American Sociological Review 52: 421-439.

Castoriadis, C., 1984: Gesellschaft als imaginäre Institution. Frankfurt: Suhrkamp.

Clarke, S., 1982: Marx, Marginalism, and Modern Sociology. London: Macmillan.

Collins, R., 1985: Jeffrey Alexander and the Search for Multidimensional Theory. Theory and Society 14: 877-892.

Durkheim, E., 1987: Schriften zur Soziologie der Erkenntnis. Hans Joas (Hrsg.), Frankfurt: Suhrkamp.

Habermas, J., 1981: Theorie des kommunikativen Handelns. 2 Bände. Frankfurt: Suhrkamp.

Hennis, W., 1987: Die Spuren Nietzsches im Werk Max Webers, S. 167-191 in W. Hennis, Max Webers Fragestellung. Tübingen: Mohr.

Honneth, A./Joas, H., 1987: War Marx ein Utilitarist? Für eine Gesellschaftstheorie jenseits des Utilitarismus. S. 148-161 in: H. Steiner (Hrsg.), Karl Marx und Friedrich Engels - ihr Einfluß und ihre Wirksamkeit in der Geschichte und Gegenwart der soziologischen Theorie. Berlin (DDR), Band 1.

Joas, H., 1984: Handlungstheorie und das Problem sozialer Ordnung. Zur „Theorie des Handelns" von Richard Münch. Kölner Zeitschrift für Soziologie und Sozialpsychologie 36: 165-172.

Parsons, T., 1937: The Structure of Social Action. New York: Free Press.

Piaget, J., 1973: Das moralische Urteil beim Kinde. Frankfurt: Suhrkamp. 
Porpora, D., 1986: A Response to Jeffrey Alexander's "Theoretical Logic in Sociology“ Concerning the Alleged Unidimensionality of Marxian Theory. Sociological Quarterly 27: 75-90.

Taylor, Ch., 1986: Philosophical Papers. 2 Bände. Cambridge: Cambridge University Press.

Toulmin, St., 1983: Kritik der kollektiven Vernunft. Frankfurt: Suhrkamp.
Turner, St., 1985: Review of Jeffrey Alexander. Philosophy of the Social Sciences 15: 77-82, 211-216, 365-368, 513-522.

Wenzel, H., 1985: Mead und Parsons: Die emergente Ordnung des sozialen Handelns. S. 26-59 in H. Joas (Hrsg.), Das Problem der Intersubjektivität. Frankfurt: Suhrkamp. 